\title{
Influence of first trimester biochemistry methodology on detection rate in screening for trisomy 21
}

\author{
Bartosz Czuba ${ }^{1}$, Dariusz Borowski², Piotr Węgrzyn ${ }^{3}$, Wojciech Cnota ${ }^{1}$, \\ Anna Kubaty ${ }^{4}$, Mirosław Wielgoś ${ }^{2}$, Krzysztof Sodowski ${ }^{1}$ \\ ${ }^{1}$ Department of Obstetrics and Gynecology in Ruda Slaska, Medical University of Silesia, Ruda Slaska, Poland \\ ${ }_{2}^{2}{ }^{\text {st }}$ Chair and Department of Obstetrics and Gynecology, Medical University of Warsaw, Poland \\ ${ }^{3}$ Department of Obstetrics and Perinatology, Medical University of Warsaw, Poland \\ ${ }^{4}$ Department of Obstetrics, Gynecology and Oncology, The Gabiel Narutowicz Hospital, Krakow, Poland
}

\begin{abstract}
Objectives: The purpose of the study was to compare detection rates (DR) of FMF-certified and non-certified biochemical tests $(B C)$ in trisomy 21 screening at 11-13+6 weeks.

Material and methods: In 2267 singleton pregnancies FMF-certified doctors measured crown to rump length (CRL) and nuchal translucency (NT). Serum samples were tested for free $\beta$-hCG and the PAPP-A using 2 analysers (Delfia - Perkin Elmer and Immulite 2000 - DPC), the results were expressed in MoM values and used for computer calculation of the risk for trisomy 21. The cut-off value for the high trisomy 21 risk was 1:300.

Results: Comparison of free $\beta$-hCG MoMs by DPC and Delfia demonstrated statistically significant differences in normal, and trisomy 21 fetuses respectively. Similarly, statistically significant differences were noted for PAPP-A MoMs. The above differences in MoMs resulted in altered sensitivity in screening for aneuploidy. The application of the FMF-certified method ensures a markedly higher DR $=74 \%$, compared to non-certified tests (64\%), both at $5 \%$ FPR.

The ROC analysis was performed in order to assess the efficacy of both tests. Results of trisomy 21 BC + NT risk scales using the Delfia and DPC methods are highly significant $(\mathrm{p}<0.0001)$, which means that their discrimination ability is $>90 \%$. The difference between results obtained using the Delfia and DPC methods is AUC $=0.0150$ and is statistically significant $(\mathrm{Z}=2.4728, \mathrm{p}=0.0134)$.

Conclusions: The use of FMF-certified first trimester biochemistry analysers improves DR for trisomy 21. The use of non-certified analysers causes reduction of DR and an increase of invasive procedure rate.

Key words: screening for chromosomal defects, 11 to $13+6$ weeks scan, trisomy 21, first trimester biochemistry, PAPP-A, free beta-hCG
\end{abstract}

Ginekologia Polska 2017; 88, 9: 492-496

\section{INTRODUCTION}

Contemporary prenatal screening is based on ultrasound examinations and biochemical tests [1]. Many professional societies and public healthcare organisation have accepted the trisomy 21 (Down syndrome) risk estimation model created by the Fetal Medicine Foundation (FMF). The FMF First Trimester Screening (FTS) scheme involves ultrasound assessment of fetal structures, biometric measurements: Crown-Rump Length (CRL) and Nuchal Trans- lucency (NT), along with first trimester biochemistry (BC) test. The latter, sometimes called "double test" is based on the levels of free $\beta$-hCG and pregnancy-associated plasma protein type A (PAPP-A) in maternal serum expressed as Multiple of the Median (MoM). BC test values and ultrasound measurements are analysed by appropriate FMF-certified computer software to calculate patient individual risk for trisomy 21, 18 and 13 respectively (www. fetalmedicine.org). 
This system assures high Detection Rate (DR) of approx. $90 \%$, with a relatively low False Positive Rate (FPR) leading to invasive testing rate of approx. 5\% [2]. The efficacy of screening may be increased, with a simultaneous reduction of invasive testing rate, by addition of ultrasound markers of trisomy 21 (NB, DV PI, TR, FMA-angle) to the risk calculation algorithm. It should be noted that the use of additional markers should only be considered when the risk based on Maternal Age (MA), NT and the biochemistry test falls within the range of 1/50-1/1000 [3-6].

Addition of the first trimester biochemistry to the screening previously based on MA and NT increased DR substantially. In trisomy 21 there is a significant increase of free $\beta$-hCG level in maternal serum during the first and the second trimester. PAPP-A level increases throughout the whole duration of uncomplicated pregnancy. In trisomy 21 PAPP-A level drops during the first trimester and in the second trimester appears normal or slightly reduced [1, 5]. Expressing free $\beta$-hCG and PAPP-A in MoMs allowed creating efficient risk calculation algorithm [7].

Maintenance of high standards of both ultrasound scan and first trimester biochemistry is crucial for the quality of screening. FMF provides training for physicians and sonographers that is concluded with a certificate for the 11-14 weeks scan. The quality of the ultrasound scan is ensured by a system of yearly auditing. There is also a system of certification for first trimester biochemistry analysers. It should be noted that at present only three biochemistry analysers have the FMF certificate (manufactured by Perkin Elmer, Brahms, Roche). This ensures high DR (approx. 90\%), with the acceptable ratio of indications for invasive testing (FPR - approx. 5\%). The use of non-certified first trimester biochemistry analysers potentially can have a detrimental effect on the quality of screening.

\section{OBJECTIVES}

The purpose of the study was to compare DRs for trisomy 21 screening at 11-14 weeks of pregnancy, based on biochemical tests certified by the FMF (Delfia Express) and non-certified by the FMF (DPC — PRISCA).

\section{MATERIAL AND METHODS}

2267 fetuses from singleton pregnancies were scanned at 11-14 weeks between September 2006 and March 2008. Physicians with valid FMF license measured CRL and NT. Ultrasound scans were performed using the Voluson Expert VE 730 equipment (General Electric). Maternal age and last menstrual period date were noted. Two blood samples, $5 \mathrm{~mL}$ each, were collected from each patient. Serum samples ( 2 from each patient) were stored at $-18^{\circ} \mathrm{C}$ and later tested for free $\beta$-hCG and the PAPP-A level; using 2 methods: immunofluorescence (delayed fluorescence - Delfia — Perkin
Elmer) and ELISA (enzyme-linked immunosorbent assay — Immulite 2000 - DPC). The results were later transformed into MoM values and used for computer calculation of the risk of trisomy 21 (FMF-certified Astraia software for Delfia and Prisca for Immulite). Considering the applied biochemical test methodology there were two study groups (DPC and Delfia).

In both groups patients with trisomy 21 risk $\geq 1: 300$ were offered an invasive test (amniocentesis) for karyotyping. If the patient declined invasive testing, the karyotype was determined after birth, if the phenotype was suspicious for Down syndrome. The trisomy 21 status was determined for all patients. Based on the karyotype, patients were divided into two groups: normal karyotype and trisomy 21 . Results were analysed with the statistical suite PQStat ver. 1.4.2.324.

The U Mann-Whitney test was used to compare the results for the trisomy 21 group with the results for the group with normal karyotype. The Wilcoxon test was used for comparison between results of Delfia and DPC.

The correlation between results of risk scores with a cut-off value of 1:300, and occurrence of trisomy 21 was analysed using the chi-squared test. The DR and the FPR were determined. Intra-class correlations were evaluated and Bland-Altman analyses were completed for risk results obtained for both methods: Delfia and DPC. ROC analyses were performed for $\mathrm{BC}$ only and $\mathrm{BC}+\mathrm{NT}$ risk scores for Delfia and DPC respectively. The test probability at the level of $p<0.05$ was accepted as significant.

\section{RESULTS}

Comparison of test parameters is presented in the Table 1. The biochemistry parameters were statistically significantly different in the Delfia and in the DPC group, in relation to the karyotype. The only parameter, for which no statistically significant difference was found, was the free $\beta$-hCG level assayed using the DPC equipment and expressed in absolute values. Figures 1 and 2 show median distribution of risk scores in the T21 and no-T21 groups.

The Bland-Altman analysis was applied in order to establish an intra-class correlation. A highly significant $(p<0.0001)$ intra-class correlations were found between results for the methods Delfia and DPC. In case of the trisomy $21 \mathrm{BC}$ risk the correlation was high $=0.895$, and in case of the trisomy $21 \mathrm{BC}+\mathrm{NT}$ risk the inter-class correlation was even higher $=0.9905$. For trisomy $21 \mathrm{BC}$ risk results the variance coefficient was approx. 40\%, and for the T21 BC + NT risk was lower $=13.29 \%$ (Figure 3).

The ROC analysis was performed in order to assess the clinical efficacy of both tests (DPC BC + NT and Delfia $B C+N T$ ) in screening for trisomy 21 (Figures 4 and 5).

In case of the trisomy $21 \mathrm{BC}$ risk scales for both the Delfia and the DPC methods, no significant $(p>0.05)$ discrimina- 
Table 1. Comparison of test parameters - normal karyotype and trisomy 21

\begin{tabular}{|c|c|c|c|c|c|c|c|}
\hline Variable & Trisomy 21 & Mean & SD & Minimum & Median & Maximum & U Mann-Whitney test \\
\hline \multirow{2}{*}{ The risk of T21 BC NT Delfia } & Yes & 518.54 & 2649.96 & 2 & 27 & 24825 & $Z=-14.7550$ \\
\hline & No & 9297 & 8608.9 & 2 & 6350 & 31399 & $p<0.0001$ \\
\hline \multirow{2}{*}{ The risk of T21 BC NT DPC } & Yes & 551.26 & 2756.89 & 2 & 27 & 24825 & $Z=-14.4523$ \\
\hline & No & 9149.5 & 8577.59 & 2 & 6287 & 31399 & $p<0.0001$ \\
\hline \multirow{2}{*}{$\beta$-hCG Delfia } & Yes & 56.59 & 32.84 & 2.3 & 51 & 167 & $Z=2.0779$ \\
\hline & No & 52.01 & 40.29 & 2.8 & 41.9 & 472.33 & $p=0.0377$ \\
\hline \multirow{2}{*}{$\beta$-hCG DPC } & Yes & 58.45 & 36.43 & 2.1 & 50 & 167 & $Z=1.5112$ \\
\hline & No & 54.29 & 41.52 & 2.8 & 43.1 & 472.33 & $p=0.1307$ \\
\hline \multirow{2}{*}{$\beta$-hCG - MoMs Delfia } & Yes & 1.67 & 0.95 & 0.2 & 1.33 & 4.36 & $Z=2.9367$ \\
\hline & No & 1.41 & 0.96 & 0.2 & 1.16 & 6 & $p=0.0033$ \\
\hline \multirow{2}{*}{$\beta$-hCG - MoMs DPC } & Yes & 1.71 & 0.92 & 0.18 & 1.58 & 4.3 & $Z=2.7562$ \\
\hline & No & 1.47 & 0.98 & 0.18 & 1.2 & 6 & $p=0.0058$ \\
\hline \multirow{2}{*}{ PAPP-A Delfia } & Yes & 9.34 & 60.77 & 0.2 & 2.76 & 59.8 & $Z=-2.8417$ \\
\hline & No & 4.11 & 4.43 & 0.31 & 3.11 & 60.45 & $p=0.0045$ \\
\hline \multirow{2}{*}{ PAPP-A DPC } & Yes & 3.2 & 2.38 & 0.19 & 2.78 & 13.1 & $Z=-2.0532$ \\
\hline & No & 4.01 & 4.37 & 0.05 & 3.07 & 60.45 & $p=0.0400$ \\
\hline \multirow{2}{*}{ PAPP-A MoMs Delfia } & Yes & 1.18 & 1.1 & 0.16 & 0.77 & 4.49 & $Z=-3.7695$ \\
\hline & No & 1.26 & 0.81 & 0.15 & 1.09 & 6 & $p=0.0002$ \\
\hline \multirow{2}{*}{ PAPP-A MoMs DPC } & Yes & 1.18 & 1.08 & 0.16 & 0.77 & 4.49 & $Z=-3.1758$ \\
\hline & No & 1.24 & 0.8 & 0.15 & 1.08 & 6 & $p=0.0015$ \\
\hline
\end{tabular}

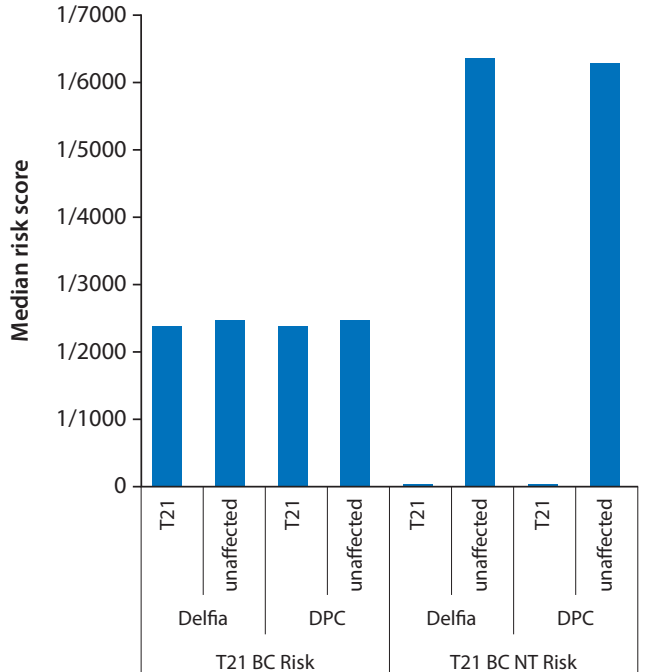

Figure 1. Median risk score distribution in the trisomy 21 (T21) and normal karyotype (unaffected) groups. It should be noted that both in the Delfia, and in the DPC group trisomy 21 risk values (calculated based on the BC $+\mathrm{NT}$ ) are significantly increased compared to the healthy controls. That difference (healthy compared to trisomy 21) was not observed for the trisomy 21 risk calculated based on the BC only for the DPC and Delfia groups

tion ability in relation to prediction of trisomy 21 was found. The difference between results obtained using the Delfia and the DPC methods is AUC $=0.0129$ and it is not signifi- cant $(Z=0.4798, p=0.6314)$. However, the differences of trisomy $21 \mathrm{BC}+\mathrm{NT}$ risk scales using the Delfia and the DPC methods are highly significant $(p<0.0001)$, which means that their discrimination ability is high — over $90 \%$. The difference between results obtained using the Delfia and the DPC methods is AUC $=0.0150$ and is statistically significant $(Z=2.4728, p=0.0134)$ (Table 2).

DR values for different FPR for DPC and Delfia for combined tests $(\mathrm{NT}+\mathrm{BC})$ are shown in Table 3.

\section{DISCUSSION}

Fetal Medicine Foundation screening for chromosomal defects with 11-14 wks scan with NT measurement and first trimester biochemistry test currently is a standard in many countries [8]. Polish Gynecological Society guidelines on prenatal diagnosis recommend application of both tests (first trimester biochemistry test and nuchal scan) at 11-14 weeks of pregnancy [9].

There are very few publications regarding PAPP-A and free $\beta$-hCG levels in the Polish population. A limited number of FMF-certified analysers could be responsible for slow progress of introduction of first trimester biochemistry testing in Poland, where ELISA tests have been long used for assaying test substances. Despite a doubtless clinical value of the latter method, its inconsistency with FMF standards has to be stressed. The only available 


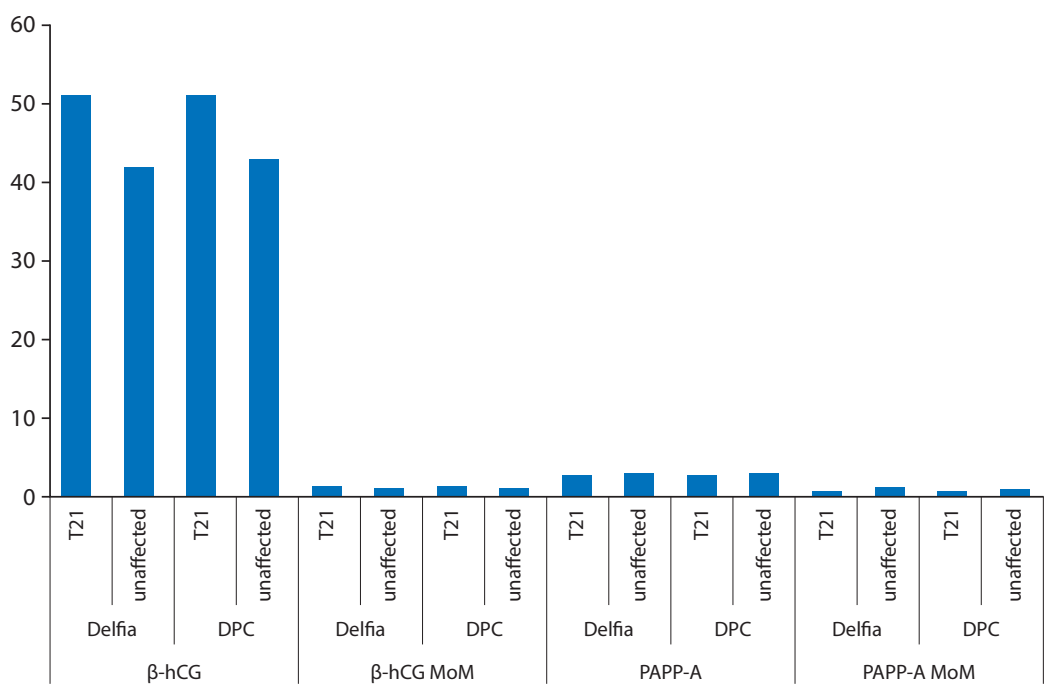

Figure 2. Median distributions of $B C$ parameters in the trisomy 21 (T21) and normal karyotype (unaffected) groups. All study parameters were statistically significantly different in both groups

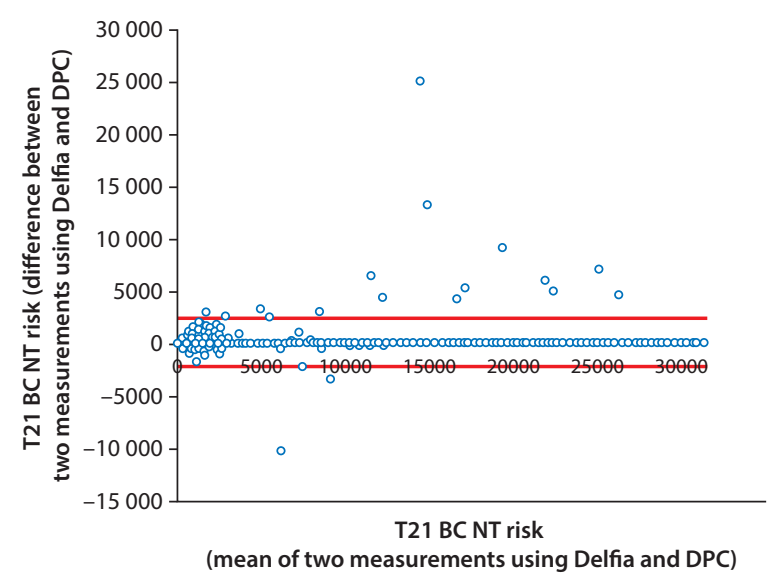

Figure 3. The Bland-Altman graph for the variable - the T21 BC + NT risk

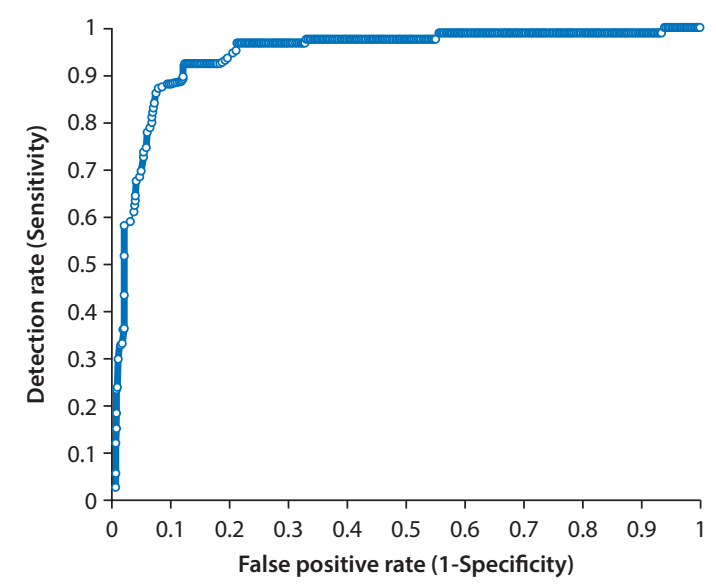

Figure 5. The ROC curve for the scale - the T21 BC + NT Delfia risk

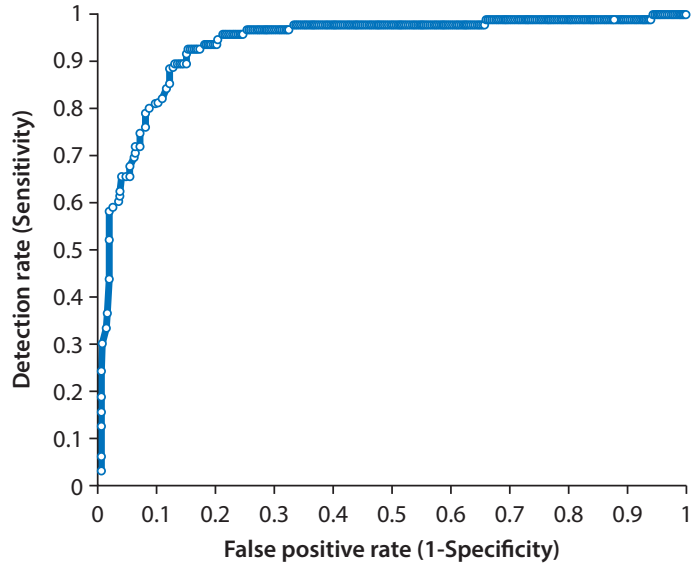

Figure 4. The ROC curve for the scale - the T21 BC + NT DPC risk

study comparing the ELISA-based method and methods FMF-certified is a paper by K. Spencer. The author proved that the use of a non FMF-certified analyser (DPC Immulite 2000) leads to different results of biochemical tests than those obtained using FMF-certified equipment [10]. The mean free $\beta$-hCG MoM value in trisomy 21 was 1.703 for the DPC, compared to 1.698 for the analysis performed using FMF-certified Kryptor (Brahms) and the difference was not statistically significant. But the mean PAPP-A value in trisomy 21 measured using DPC was $0.62 \mathrm{MoM}$, compared to $0.47 \mathrm{MoM}$ in case of the analysis by Kryptor, and that difference was statistically significant $(p=0.025)$. Authors noticed that this affects sensitivity of the screening for trisomy 21. Screening based on DPC equipment ensured DR $=58 \%$ 
Table 2. The ROC analysis of the risk of trisomy 21 (T21)

\begin{tabular}{|l|c|c|c|c|c|c|}
\hline & AUC & SE (AUC) & $-95 \%$ Cl & $+95 \%$ Cl & $\mathbf{Z}$ & $\mathbf{p}$ \\
\hline The risk of T21 BC Delfia & 0.5298 & 0.0358 & 0.4596 & 0.6000 & 1.00 & 0.3159 \\
\hline The risk of T21 BC DPC & 0.5168 & 0.0345 & 0.4491 & 0.5845 & 0.57 & 0.5708 \\
\hline The risk of T21 BC + NT Delfia & 0.9411 & 0.0124 & 0.9168 & 0.9654 & 14.87 & $<0.0001$ \\
\hline The risk of T21 BC + NT DPC & 0.9262 & 0.0142 & 0.8983 & 0.9540 & 14.36 & $<0.0001$ \\
\hline
\end{tabular}

Table 3. DR values for different FPR values for both methods (DPC and Delfia) in combined test (NT + BC)

\begin{tabular}{|l|l|l|l|}
\hline FPR & $\mathbf{5} \%$ & $\mathbf{1 0} \%$ & $\mathbf{1 5} \%$ \\
\hline DR for Delfia & $74 \%$ & $88 \%$ & $92 \%$ \\
\hline DR for DPC & $64 \%$ & $79 \%$ & $87 \%$ \\
\hline
\end{tabular}

with a $5 \%$ FPR, compared to DR $=62 \%$ for FMF-certified Kryptor analyser [10].

Similar results were obtained in our study. The free $\beta$-hCG DPC MoM range was 0.18-4.3, and free $\beta$-hCG Delfia MoM was $0.2-4.36$ in normal babies. In trisomy 21 the range of free $\beta$-hCG DPC MoM was 0.18-6, and free $\beta$-hCG Delfia MoM was $0.2-6$. Statistically significant differences were demonstrated for $\beta$-hCG MoM, and no such difference was found for the free $\beta$-hCG level expressed in absolute values. Similar statistically significant differences were noted for PAPP-A levels. The PAPP-A DPC MoM range for was (0.15-6) in trisomy 21 patients, and was the same in the PAPP-A Delfia MoM. For normal fetuses the PAPP-A MoM ranges for DPC and Delfia were identical: 0.16-4.49.

In our study the differences between both methods in $B C$ results expressed in MoMs significantly influenced sensitivity of screening for chromosomal defects, similarly to the study by Spencer et al. [10]. DRs for trisomy 21 at various FPR are presented in the Table 3 . Those results clearly indicate that in each case the application of the FMF-certified method (Delfia) ensures a markedly higher DR compared to non-certified tests (DPC). At 5\% FPR in the FMF-certified method the DR is $10 \%$ better.

ROC graphs illustrate that both test methods offer significant discrimination abilities, with some preponderance of the FMF-certified.

The FMF-certified BC test provides a significantly higher DR for trisomy 21, with a potentially lower FPR; hence lower number of invasive procedures.

\section{CONCLUSIONS}

The use of FMF-certified analysers for the first trimester biochemistry in the combined test improves DR in screening for trisomy 21.

The use of non-certified analysers for first trimester biochemistry decreases DR of the combined test and causes an increased invasive procedures rate.

\section{REFERENCES}

1. Nicolaides K. Screening for fetal aneuploidies at 11 to 13 weeks. Prenatal Diagnosis. 2011; 31(1): 7-15, doi: 10.1002/pd.2637.

2. Nicolaides $\mathrm{KH}$. A model for a new pyramid of prenatal care based on the 11 to 13 weeks' assessment. Prenat Diagn. 2011; 31(1): 3-6, doi: 10.1002/pd.2685, indexed in Pubmed: 21210474.

3. Kagan KO, Cicero S, Staboulidou I, et al. Fetal nasal bone in screening for trisomies 21, 18 and 13 and Turner syndrome at 11-13 weeks of gestation. Ultrasound Obstet Gynecol. 2009; 33(3): 259-264, doi: 10.1002/uog.6318, indexed in Pubmed: 19248005.

4. Kagan KO, Valencia C, Livanos $\mathrm{P}$, et al. Tricuspid regurgitation in screening for trisomies 21,18 and 13 and Turner syndrome at $11+0$ to $13+6$ weeks of gestation. Ultrasound Obstet Gynecol. 2009; 33(1): 18-22, doi: 10.1002/uog.6264, indexed in Pubmed: 19031473.

5. Wright D, Syngelaki A, Bradbury I, et al. First-trimester screening for trisomies 21,18 and 13 by ultrasound and biochemical testing. Fetal Diagn Ther. 2014; 35(2): 118-126, doi: 10.1159/000357430, indexed in Pubmed: 24356462.

6. Czuba B, Cnota W, Wloch A, et al. Frontomaxillary facial angle measurement in screening for trisomy 18 at $11+0$ to $13+6$ weeks of pregnancy: a double-centre study. Biomed Res Int. 2013; 2013: 168302, doi: 10.1155/2013/168302, indexed in Pubmed: 24195065.

7. Noble PL, Abraha HD, Snijders RJ, et al. Screening for fetal trisomy 21 in the first trimester of pregnancy: maternal serum free beta-hCG and fetal nuchal translucency thickness. Ultrasound Obstet Gynecol. 1995; 6(6): 390-395, doi: 10.1046/j.1469-0705.1995.06060390.x, indexed in Pubmed: 8903913.

8. Spencer K, Souter V, Tul N, et al. A screening program for trisomy 21 at 10-14 weeks using fetal nuchal translucency, maternal serum free beta-human chorionic gonadotropin and pregnancy-associated plasma protein-A. Ultrasound Obstet Gynecol. 1999; 13(4): 231-237, doi: 10.1046/j.1469-0705.1999.13040231.x, indexed in Pubmed: 10341399.

9. Pietryga M, Borowski D, Czekierdowski A, et al. Polish Gynecological Society Ultrasound Section Guidelines on ultrasound screening in uncomplicated pregnancy. Ginekol Pol. 2012; 83(4): 309-315.

10. Spencer K. First trimester maternal serum screening for Down's syndrome: an evaluation of the DPC Immulite 2000 free beta-hCG and pregnancy-associated plasma protein-A assays. Ann Clin Biochem. 2005; 42(Pt 1): 30-40, doi: 10.1258/0004563053026880, indexed in Pubmed: 15802030. 